\title{
Effects of disopyramide on electrophysiological properties of specialized conduction system in man and on accessory atrioventricular pathway in Wolff-Parkinson-White syndrome
}

\author{
R. A. J. Spurrell, C. W. Thorburn, J. Camm, E. Sowton, and D.'C.'Deuchar \\ From the Departments of Cardiology at St. Bartholomew's Hospital and Guy's Hospital, London
}

Seven patients with normal specialized conduction system and three patients with the Wolff-Parkinson-White (WPW) syndrome were studied using programmed stimulation of the heart before and after the administration of intravenous disopyramide. The principal effect of this drug was to prolong the effective refractory period of the atria and ventricles, and to prolong the effective refractory period of the anomalous pathway in the WPW syndrome. In addition, it prolonged the conduction time in the anomalous pathway in the WPW syndrome. These findings suggest that disopyramide would be a useful and safe drug in the management of certain atrial and ventricular arrhythmias and in the management of the Wolff-Parkinson-White syndrome with atrial fibrillation.

Disopyramide (Rythmodan) is an antiarrhythmic agent which has been found valuable in the management of extrasystoles and tachycardias of both supraventricular and ventricular origin (Vismara, Mason, and Amsterdam, 1974; Katz et al., 1963; Desruelles et al., 1967). Using techniques of intracardiac recordings and programmed electrical stimulation of the heart, we have studied the effects of disopyramide on the electrophysiological properties of the specialized conduction system in man, and in addition its effect on the anomalous atrioventricular pathway in the Wolff-Parkinson-White (WPW) syndrome.

\section{Patients and methods}

Ten patients, 5 male and 5 female, were studied and their clinical details are shown on Table I. Case I underwent an electrophysiological study while undergoing routine cardiac catheterization for a ventricular septal defect. Cases 2, 4, 5, and 6 were undergoing routine electrophysiological study for the investigation of recurrent rapid palpitations. In each patient the resting electrocardiogram was normal and no tachycardia could be initiated during the study which in each patient demonstrated a normal conduction system. Cases 3 and 7 underwent electrophysiological study for the further investigation of previously documented ventricular Received 17 March 1975. tachycardia: in both patients the resting electrocardiograms were normal. Cases 8, 9, and to had the WPW syndrome with recurrent bouts of supraventricular tachycardia; the resting electrocardiogram in each patient showed the typical features of the WPW syndrome. Informed consent was obtained from each patient or from the parents of Cases 1,8 , and 9. All patients underwent the electrophysiological study in the postabsorptive non-sedated state. No patient was receiving drug medication at the time of the study. Three bipolar electrode catheters were introduced percutaneously via one or both femoral veins. One catheter was positioned high in the right atrium to record a high right atrial electrogram. One catheter was positioned across the tricuspid valve to record a His bundle electrogram using the technique described by Scherlag et al. (1969). The His bundle electrogram was recorded at a filter setting of 40 to $50 \mathrm{c} / \mathrm{s}$. The intracardiac recordings were made simultaneously with leads I, III, VI, and V6 of the surface electrocardiogram. All recordings were made on an 8-channel Elema Mingograph 8I at a paper speed of $100 \mathrm{~mm} / \mathrm{s}$. The third catheter was positioned in either the right atrium or ventricle for atrial or ventricular pacing and inducing single or double atrial or ventricular premature beats. In Cases 8 and ro an additional catheter electrode was positioned in the coronary sinus to record left atrial activity.

A Devices $4270^{1}$ stimulator was used for atrial and ${ }^{1}$ Devices Instruments Ltd., Welwyn Garden City, Hertfordshire. 
TABLE I Clinical details

\begin{tabular}{rlll}
\hline $\begin{array}{l}\text { Case } \\
\text { No. }\end{array}$ & Age & Sex & Clinical diagnosis \\
\hline I & I7 & M & VSD \\
2 & 30 & F & Paroxysmal tachycardia \\
3 & 30 & F & Paroxysmal ventricular tachycardia \\
4 & 49 & F & Paroxysmal tachycardia \\
5 & 40 & F & Paroxysmal tachycardia \\
6 & 33 & M & Paroxysmal tachycardia \\
7 & 32 & M & Paroxysmal ventricular tachycardia \\
8 & I5 & F & Type A WPW syndrome \\
9 & II & M & Type B WPW syndrome \\
I0 & 34 & M & Type A WPW syndrome \\
\hline
\end{tabular}

ventricular pacing. The heart was driven at a constant frequency to avoid changes in excitability or refractoriness caused by variations in rate or rhythm. Single or double atrial or ventricular premature beats were delivered at a preset time in the cardiac cycle, after every eighth beat of the basic driven rhythm.

The following measurements were made.

$S t_{1}-S t_{2}$ : time in milliseconds between two successive pacing stimuli.

$H R A-L R A$ : time in milliseconds from the high right atrial potential as recorded by the electrode catheter high in the right atrium to the low right atrial potential as seen on the His bundle electrogram. The atrial pacing electrode was usually positioned at the superior vena cava-right atrial junction in order to stimulate the atria in the region of the sinoatrial node.

$L R A-H$ : time in milliseconds from the atrial potential on the His bundle electrogram to the His potential on the His bundle electrogram. This measurement represents atrioventricular nodal conduction.

$H-V$ : time in milliseconds from the His potential to the earliest onset of ventricular activation as seen on the surface electrogram leads.

St-d: time in milliseconds from the pacing stimulus artefact to the onset of the delta wave on the surface electrocardiogram in the patients with the WPW syndrome.

$V-A^{\prime}:$ retrograde conduction time in milliseconds in the anomalous pathway during reciprocal tachycardia from the onset of ventricular activation on the surface electrocardiogram to the earliest onset of atrial activation as seen on the right atrial electrogram (HRAE) in Case 9 (Type B WPW) and the earliest onset of atrial activation as seen on the coronary sinus electrogram in Cases 8 and ro (Type A WPW).

$A^{\prime}-V$ : antegrade conduction time in milliseconds in the atrioventricular node His pathway during reciprocal tachycardia measured from the earliest onset of atrial activation as recorded either on the right atrial electrogram (Case 9) or coronary sinus electrogram (Cases 8 and ro).

$R-R$ : cycle length of the tachycardia in milliseconds.
ERP atr: effective refractory period of atrium is the longest $\mathrm{St}_{1}-\mathrm{St}_{2}$ interval that does not result in atrial depolarization by $\mathrm{St}_{2}$.

FRP AVN: the functional refractory period of the atrioventricular node is the shortest $\mathrm{H}_{1} \mathrm{H}_{2}$ interval resulting from two conducted atrial depolarizations.

ERP AVN: the effective refractory period of the atrioventricular node is the longest $L R A_{1}-L_{R A}$ interval that does not result in conduction of $\mathrm{LRA}_{2}$ to the His bundle.

ERP ventricle: effective refractory period of the ventricle is the longest $\mathrm{St}_{1}-\mathrm{St}_{2}$ interval during ventricular premature stimulation at which $\mathrm{St}_{2}$ fails to produce a ventricular depolarization.

ERP anomalous bypass (antegrade or retrograde): antegrade or retrograde effective refractory period of the anomalous bypass is the longest atrial or ventricular premature beat interval at which the anomalous pathway failed to conduct either antegradely or retrogradely. The criteria for conduction occurring retrogradely in an anomalous bypass have been described previously by Wellens and Durrer (1974).

Latency time is the time from the pacing stimulus artefact to the earliest onset of atrial or ventricular activation. All measurements were made in milliseconds.

The above measurements were made before the administration of disopyramide and were repeated at 2,5 , 10, 15, 20, and 30 minutes after the intravenous administration of disopyramide in a dose of $2 \mathrm{mg} / \mathrm{kg}$ body weight. In the patients with the WPW syndrome the tachycardia was initiated before the administration of the drug in each case in order to study the effect of the drug on the reciprocal circuit during tachycardia.

Only the maximal changes in the conduction times or refractory periods after disopyramide administration are reported in the Tables and in each patient this occurred between 2 and 5 minutes after administration. In most patients all the measurements had returned to near control values after 30 minutes.

The conduction times before and after disopyramide are given at the same basic $\mathrm{St}_{1}-\mathrm{St}_{2}$ intervals to avoid changes in conduction caused by changes in cycle length. For the same reason refractory periods were recorded before and after disopyramide using the same basic cycle during premature stimulation.

\section{Results}

No patient suffered any side effect from the administration of disopyramide.

Patients with normal conduction system

Table 2 summarizes the effects of this drug on intra-atrial conduction (HRA-LRA), atrioventricular nodal conduction (LRA-H), and His Purkinje conduction (H-V) in Cases $\mathrm{I}$ to 7 . In 4 patients the HRA-LRA interval increased by $10 \mathrm{~ms}$ suggesting an increase in atrial conduction time. Small changes in LRA-H time were noted. In 5 patients, the conduction time increased from between 5 and $15 \mathrm{~ms}$, in one patient there was a $10 \mathrm{~ms}$ 
TABLE 2 Intra-atrial, atrioventricular nodal, and intraventricular conduction times (ms) before and after disopyramide

\begin{tabular}{lllrrlllll}
\hline Case No. & \multicolumn{2}{l}{$\begin{array}{l}\text { HRA-LRA } \\
\text { Before }\end{array}$} & After & $\begin{array}{l}L R A-H \\
\text { Before }\end{array}$ & After & $\begin{array}{l}H-V \\
\text { Before }\end{array}$ & After & $\begin{array}{l}S t_{1}-S t_{2} \\
\text { Before }\end{array}$ & After \\
\hline I & 50 & 60 & 60 & 70 & 50 & 50 & 570 & 570 \\
2 & 50 & 60 & 120 & 135 & 35 & 35 & 570 & 570 \\
3 & 30 & 30 & 60 & 70 & 35 & 55 & 600 & 600 \\
4 & 60 & 60 & 100 & 100 & 35 & 40 & 600 & 600 \\
5 & 60 & 70 & 60 & 70 & 35 & 35 & 600 & 600 \\
6 & 50 & 50 & 120 & 110 & 50 & 50 & 600 & 600 \\
7 & 60 & 70 & 125 & 130 & 50 & 55 & 600 & 600 \\
\hline
\end{tabular}

TABLE 3 Atrial, atrioventricular nodal, and ventricular refractory periods (ms) before and after disopyramide

\begin{tabular}{|c|c|c|c|c|c|c|c|c|}
\hline \multirow{2}{*}{ Case No. } & \multirow{2}{*}{$\begin{array}{l}\text { ERP atr } \\
\text { Before }\end{array}$} & \multirow{2}{*}{ After } & \multirow{2}{*}{\multicolumn{2}{|c|}{$\begin{array}{l}\text { FRP } A V N \\
\text { Before } \quad \text { After }\end{array}$}} & \multicolumn{2}{|c|}{$E R P A V N$} & \multicolumn{2}{|c|}{ ERP ventricle } \\
\hline & & & & & Before & After & Before & After \\
\hline $\mathbf{I}$ & 230 & 270 & 400 & 400 & $<230$ & $<270$ & 170 & 190 \\
\hline 2 & 210 & 280 & 450 & 450 & 270 & 290 & 220 & 230 \\
\hline 3 & 250 & 365 & 450 & 450 & 280 & $<365$ & 320 & 370 \\
\hline 4 & 270 & 290 & 560 & 550 & 320 & 320 & 200 & 230 \\
\hline 5 & 240 & 290 & 450 & 450 & 260 & $<290$ & 210 & 230 \\
\hline 6 & 190 & 270 & 430 & 450 & 300 & 300 & 170 & 185 \\
\hline 7 & 235 & 270 & 460 & 460 & 345 & 350 & 240 & 255 \\
\hline
\end{tabular}

decrease, and in one patient there was no change. Intraventricular conduction $(\mathrm{H}-\mathrm{V})$ was unchanged in 4 patients, increased by only $5 \mathrm{~ms}$ in 2 patients, and increased by $20 \mathrm{~ms}$ in I patient; this latter patient (Case 3) had a normal $\mathrm{H}-\mathrm{V}$ time before disopyramide administration.

Table 3 summarizes the results of the measurements of the refractory periods in Cases I to 7. The effective refractory period of the atria was increased in each patient by between 20 and $115 \mathrm{~ms}$.

The functional refractory period of the atrioventricular node was minimally changed in 2 patients only.

The effective refractory period of the atrioventricular node could not be measured either before or after disopyramide in Case I owing to the effective refractory period of the atria being longer than that of the atrioventricular node. In Cases 3 and 5 the effective refractory period of the atrium was increased by disopyramide to such an extent that it became longer than that of the atrioventricular node and as such the effective refractory period of the atrioventricular node could not be measured in these 2 patients after disopyramide administration. In the remaining patients the effective refractory period of the atrioventricular node was only minimally affected in that it increased by $5 \mathrm{~ms}$ in one patient, by $20 \mathrm{~ms}$ in another, and was unchanged in 2 patients.

The effective refractory period of the ventricle was consistently increased by between 10 and $50 \mathrm{~ms}$ in all 7 patients after the administration of disopyramide.

During refractory period measurements it was found that after disopyramide administration the latency time increased in some patients, particularly during premature stimulation.

\section{Patients with WPW syndrome}

Table 4 summarizes the effects of disopyramide on the antegrade conduction time in the accessory pathway as represented by the St-d interval, in the

TABLE 4 Antegrade conduction times (ms) in the accessory pathway in WPW syndrome before and after disopyramide

\begin{tabular}{lcll}
\hline $\begin{array}{l}\text { Case } \\
\text { No. }\end{array}$ & $\begin{array}{l}\text { St-d } \\
\text { Before }\end{array}$ & After & $S t_{1}-S t_{2}$ \\
\hline 8 & 145 & $\begin{array}{l}\text { 210 } \\
\text { Bypass } \\
\text { blocked }\end{array}$ & 600 \\
9 & 120 & 110 & 600 \\
10 & 70 & & \\
\hline
\end{tabular}


864 Spurrell, Thorburn, Camm, Sowton, and Deuchar

TABLE 5 Antegrade and retrograde effective refractory period (ms) of the accessory pathway before and after disopyramide

\begin{tabular}{lllll}
\hline Case No. & \multicolumn{2}{c}{$\begin{array}{l}\text { ERP anomalous bypass antegrade } \\
\text { Before }\end{array}$} & After & \multicolumn{2}{c}{$\begin{array}{c}\text { ERP anomalous bypass retrograde } \\
\text { Before }\end{array}$} & After \\
\hline 8 & 350 & 400 & 290 & 330 \\
9 & 290 & Bypass & 280 & $\begin{array}{c}\text { Bypass } \\
\text { blocked }\end{array}$ \\
& & blocked & 270 & 310 \\
\hline
\end{tabular}

TABLE 6 Antegrade and retrograde conduction times (ms) during supraventricular tachycardia before and after disopyramide

\begin{tabular}{rllllll}
\hline Case No. & $\begin{array}{l}V-A^{\prime} \\
\text { Before }\end{array}$ & After & $\begin{array}{l}A^{\prime}-V \\
\text { Before }\end{array}$ & After & $\begin{array}{l}R-R \\
\text { Before }\end{array}$ & After \\
\hline 8 & 130 & 170 & 165 & 190 & 295 & 360 \\
9 & 120 & 170 & 180 & 130 & 300 & 300 \\
10 & 170 & 215 & 230 & 135 & 400 & 350 \\
\hline
\end{tabular}
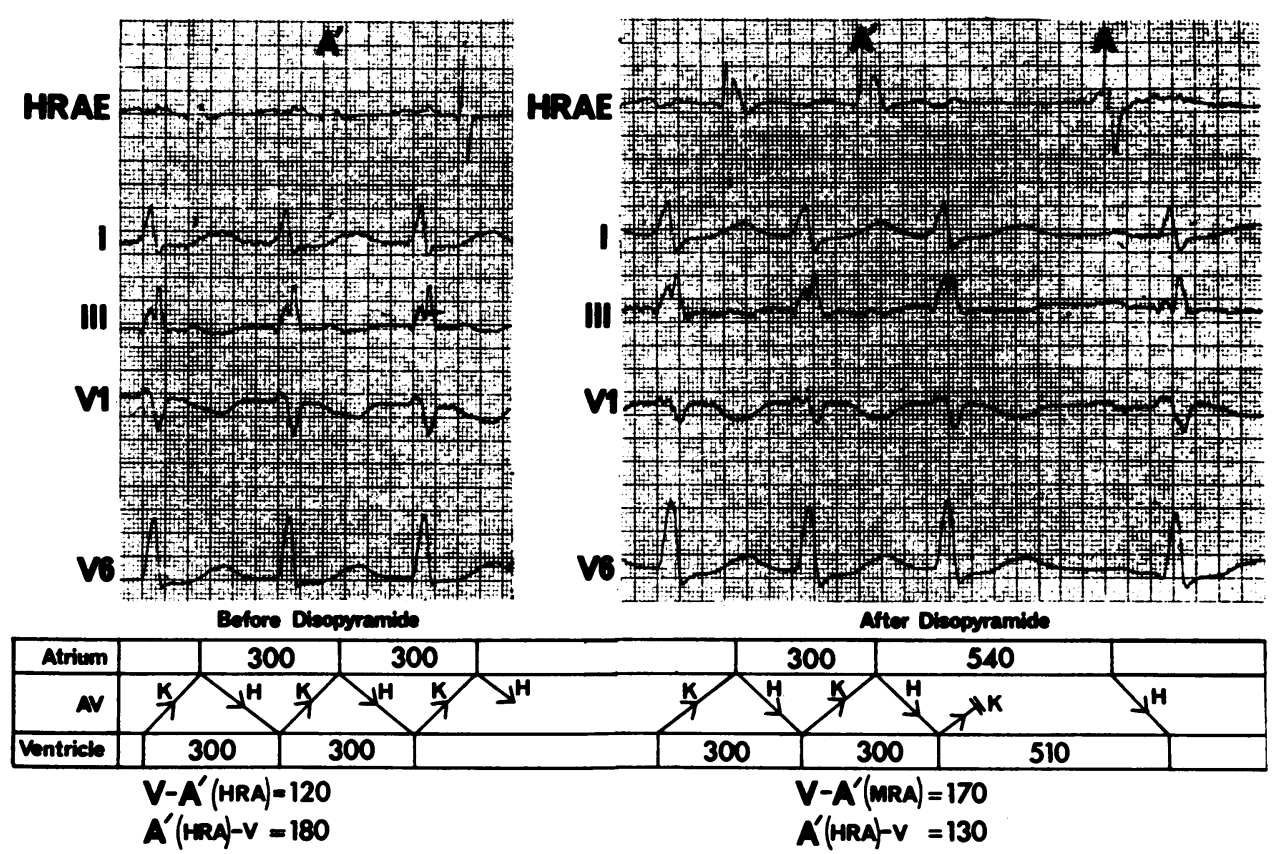

FI G. Recording obtained from Case 9 during tachycardia (left-hand panel) and after disopyramide administration (right-hand panel). In the right-hand panel the first three beats are the last three of the tachycardia and the fourth beat is the first beat of sinus rhythm. HRAE=high right atrial electrogram; I, III, VI, and V6 = surface electrocardiogram leads; $A V=$ atrioventricular region; $A^{\prime}=$ retrograde atrial potential as seen on the $H R A E ; K=K e n t$ accessory pathway; $H=$ atrioventricular node $H$ is pathway; $V-A^{\prime}(H R A)=$ retrograde conduction time ( $m s)$ from the onset of ventricular activation as seen on the surface electrocardiogram to the retrograde atrial potential as seen on the HRAE; $A^{\prime}(H R A)-V=$ antegrade conduction time (ms) from the retrograde atrial potential as seen on the HRAE to the onset of ventricular activation as seen on the surface electrocardiogram. 
3 patients with the WPW syndrome. It can be seen that the conduction time increased from between 40 and $65 \mathrm{~ms}$ in Cases 8 and $\mathrm{ro}$ and in Case 9 antegrade conduction in the accessory pathway was totally blocked and did not return until 40 minutes after disopyramide administration.

Table 5 summarizes the effects of disopyramide on the effective refractory periods of the accessory pathway for both antegrade and retrograde conduction. In Case 9 the accessory pathway was totally blocked both antegradely and retrogradely and so the refractory period could not be measured. In Cases 8 and ro the effective refractory period of the anomalous pathway for antegrade conduction increased by $50 \mathrm{~ms}$ and $65 \mathrm{~ms}$, respectively, and for retrograde conduction by $40 \mathrm{~ms}$ in both patients.

Table 6 summarizes the effect of disopyramide on retrograde conduction and antegrade conduction during reciprocal tachycardia. After disopyramide the retrograde conduction time in the anomalous bypass was prolonged in Cases 8, 9, and 10 by 40, 50 , and $45 \mathrm{~ms}$, respectively. The antegrade conduction time in the atrioventricular node His pathway was prolonged in Case 8 by $25 \mathrm{~ms}$ but shortened in Cases 9 and Io by $50 \mathrm{~ms}$ and $95 \mathrm{~ms}$, respectively. The net result was to lengthen the cycle length of the tachycardia in Case 8, the cycle length of the tachycardia shortened by $50 \mathrm{~ms}$ after disopyramide in Case IO, and the cycle length was unchanged in Case 9 (see Fig. which shows the termination of a supraventricular tachycardia in Case 9 after disopyramide administration). After the administration of disopyramide the tachycardia was terminated after 2 minutes and $2 \frac{1}{2}$ minutes, respectively, in Cases 9 and ro and the antegrade and retrograde conduction times were measured during the last cycle of the tachycardia before termination. In Case 8 the tachycardia was not terminated by disopyramide and the antegrade and retrograde conduction times were measured two minutes after the administration of disopyramide, at which time maximum prolongation of the retrograde conduction time had occurred. In Case 8 the tachycardia was finally terminated by an appropriately timed right ventricular premature beat.

\section{Discussion}

Disopyramide is an antiarrhythmic agent with an antiarrhythmic spectrum similar to that of quinidine. In the electrophysiological study presented in this paper the conduction times in the atria, atrioventricular node, and His Purkinje system show only small changes except in one patient who had a $20 \mathrm{~ms}$ increase in intraventricular conduction time. Quinidine therefore differs in that it is known to depress His Purkinje conduction (Damato and Lau, I970).

The refractory period measurements in Cases $I$ to 7 showed that the main effect of disopyramide was to increase the effective refractory period of the atria, in one patient by as much as II $5 \mathrm{~ms}$. Similar results were also obtained by Josephson et al. (1973), though the prolongation of atrial refractory period in their cases was not so striking.

In this study a consistent prolongation of the effective refractory period of the ventricle was found but was not so obvious as the prolongation of atrial refractory period.

The effect of disopyramide on the effective refractory period of the atrioventricular node was small and could not be measured in 3 patients as the effective refractory period of the atria was prolonged in excess of that of the atrioventricular node.

This predominant effect of disopyramide on the atria and ventricles may explain its apparent effectiveness in the treatment of atrial and ventricular extrasystoles (Vismara et al., 1974; Katz et al., 1963; Desruelles et al., 1967). Supraventricular tachycardias which are caused by a re-entry or reciprocal mechanism within the atria or within the atrioventricular node may be initiated by atrial extrasystoles; the effect of disopyramide on the atria and its reported effectiveness in the suppression of atrial extrasystoles should, therefore, make this a valuable drug in the prevention of paroxysms of supraventricular tachycardia caused by the above two mechanisms. In terms of termination rather than prevention of a supraventricular tachycardia, if the tachycardia is caused by a re-entry mechanism within the atria the prolongation of the effective refractory period of the atria by disopyramide should make this a useful drug for the termination of such tachycardias. However, because of disopyramide's minimal effect upon the atrioventricular node it would not be expected to be so effective in terminating a supraventricular tachycardia caused by an intra-atrioventricular nodal reciprocal mechanism.

The prolongation of atrial effective refractory period by disopyramide may well explain the mechanism by which this drug is reported to reduce the incidence of recurrence of atrial fibrillation after successful electroconversion to sinus rhythm (Härtel, Louhija, and Konttinen, 1974).

The prolongation of the effective refractory period of the ventricles may explain why disopyramide is effective in the suppression of ventricular ectopics. It is now well recognized that ventricular tachycardia in some patients may well be caused by a reentry or reciprocal mechanism (Wellens, Schuilenburg, and Durrer, 1972; Spurrell, Sowton, and 
Deuchar, 1973). The site of the re-entry mechanism in these patients is uncertain; it may be in the main bundle-branches, the distal His Purkinje tissue, ordinary ventricular myocardium, or combinations of these three. In this paper it has been shown that disopyramide prolongs the effective refractory period of the ventricle, and Josephson et al. (1973) have shown that disopyramide prolongs the effective refractory period of the His Purkinje system: it would, therefore, be reasonable to expect disopyramide to be of value in the termination of reentry ventricular tachycardia when the re-entry circuit is in one of the above sites.

Two main types of tachycardia occur in the WPW syndrome. Firstly, there is the circus movement supraventricular tachycardia which involves antegrade conduction by way of the atrioventricular node His pathway and retrograde conduction by way of the accessory pathway. Any therapeutic agent which is able to block one part of the reciprocal circuit may be useful in terminating tachycardias of this sort. Drugs which act predominantly on the atrioventricular node such as digoxin or verapamil (Roy, Spurrell, and Sowton, 1974) are useful drugs for their blocking action on the antegrade pathway of the tachycardia. In this study disopyramide has been shown to delay conduction and prolong the effective refractory period of the anomalous bypass in the WPW syndrome and so should be of value in the termination of the circus movement tachycardia, and indeed the tachycardia was terminated in Cases 9 and ro (Fig.). In these two patients the retrograde conduction time in the accessory pathway lengthened after disopyramide but the antegrade conduction time in the atrioventricular node His pathway shortened. In Case Io this shortening of antegrade conduction time was in fact sufficient to increase the rate of the tachycardia (the cycle length was reduced from 400 to $350 \mathrm{~ms}$ ). The reason for this shortening of antegrade conduction is not known as the studies in Cases $I$ to 7 did not suggest such an effect on the atrioventricular node His pathway. It may be that during reciprocal tachycardia the prolongation of conduction time in the retrograde part of the circuit was sufficient to allow longer for the atrioventricular nodal $\mathrm{His}$ pathway to recover and result in a faster atrioventricular nodal conduction time. In Case 8, however, disopyramide prolonged both retrograde and antegrade conduction times and it can only be assumed that this must have resulted from a direct effect by the drug on the atrioventricular node (the $\mathrm{H}-\mathrm{V}$ time was unchanged). This finding is a little bizarre as in Cases $I$ to 7 no such prolongation of atrioventricular nodal conduction by disopyramide was observed. The tachycardia was not terminated by the drug in Case 8 and this may be because disopyramide had prolonged the conduction times in both parts of the re-entry circuit, thereby allowing a balance of conduction time and refractoriness to remain between antegrade conduction in the atrioventricular node His pathway and retrograde conduction in the anomalous pathway such that the tachycardia could continue but with a longer cycle length.

The other type of tachycardia associated with the WPW syndrome is atrial fibrillation or atrial flutter. In the absence of an anomalous pathway the ventricles are protected from rapid activation during atrial fibrillation by the development of physiological block within the atrioventricular node. However, in the WPW syndrome ventricular activation may occur by way of the anomalous bypass and the frequency will depend upon the effective refractory period of the anomalous bypass. If the refractory period of the accessory pathway is too short, then there is a risk of ventricular fibrillation caused by activation of the ventricles at high frequency via the anomalous pathway from the fibrillating atria. Therefore, any drug that shortens the refractory period of the anomalous pathway will increase the risk of ventricular fibrillation when the refractory period of the bypass tract is already short. This situation has been described by Dreifus et al. (197r) in a patient with the WPW syndrome and atrial fibrillation, in whom recurrent bouts of ventricular fibrillation appeared to be related to the administration of digoxin. There is evidence that in some cases of the WPW syndrome digoxin shortens the refractory period of the anomalous pathway (Wellens and Durrer, 1973) and this could explain the above phenomenon. It is, therefore, important to assess the electrophysiological properties of a drug whose use is going to be considered in the WPW syndrome. In this paper disopyramide has been shown to prolong the effective refractory period of the anomalous bypass and so should be a safe and useful drug to administer to patients with the WPW syndrome and atrial fibrillation. In this context, therefore, administration of disopyramide would be expected to reduce the ventricular rate during atrial fibrillation.

This work was supported by a grant from Roussel Laboratories Ltd.

\section{References}

Damato, A. N., and Lau, S. H. (1970). Clinical value of the electrogram of the conduction system. Progress in Cardiovascular Diseases, 13, 119.

Desruelles, J., Gerard, A., Ducatillon, P., and Herbaux, A. (1967). Nos premiers essaes clinique du disopyramide (H 3292) dans les troubles du rhythme cardique. Thérapie, 22, 937 . 
Dreifus, L. S., Haiat, R., Watanabe, Y., Arriaga, J., and Reitman, N. (1971). Ventricular fibrillation: a possible mechanism of sudden death in patients with WolffParkinson-White syndrome. Circulation, 43, 520.

Härtel, G., Louhija, A., and Konttinen, A. (1974). Disopyramide in the prevention of recurrence of atrial fibrillation after electroconversion. Clinical Pharmacology and Therapeutics, 15, 551.

Josephson, M. E., Caracta, A. R., Lau, S. H., Gallagher, J. J., and Damato, A. N. (I973). Electrophysiological evaluation of disopyramide in man. American Heart fournal, 86, $77 \mathrm{I}$.

Katz, M. J., Meyer, C. E., El-Etr, A., and Slodki, S. J. (I963). Clinical evaluation of a new anti-arrhythmic agent, SC7031. Current Therapeutic Research, 5, 343.

Roy, P. R., Spurrell, R. A. J., and Sowton, E. (1974). The effect of verapamil on the cardiac conduction system in man. Postgraduate Medical fournal, 50, 270.

Scherlag, B. J., Lau, S. H., Helfant, R. H., Berkowitz, W. D., Stein, E., and Damato, A. N. (1969). Catheter technique for recording His bundle activity in man. Circulation, 39, 13.

Spurrell, R. A. J., Sowton, E., and Deuchar, D. C. (1973). Ventricular tachycardia in four patients evaluated by pro- grammed electrical stimulation of heart and treated in 2 patients by surgical division of anterior radiation of left bundle-branch. British Heart fournal, 35, ror4.

Vismara, L. A., Mason, D. T., and Armstrong, E. A. (1974). Disopyramide phosphate: clinical efficacy of a new oral antiarrhythmic drug. Clinical Pharmacology and Therapeutics, 16, 330.

Wellens, H. J., and Durrer, D. (1973). Effect of digitalis on atrioventricular conduction and circus-movement tachycardia in patients with Wolff-Parkinson-White syndrome. Circulation, 47, 1229.

Wellens, H. J. J., and Durrer, D. (1974). Patterns of ventriculo-atrial conduction in the Wolff-Parkinson-White syndrome. Circulation, 49, 22.

Wellens, H. J., Schuilenburg, R. M., and Durrer, D. (1972). Electrical stimulation of the heart in patients with ventricular tachycardia. Circulation, 46, 216.

Requests for reprints to Dr. R. A. J. Spurrell, Department of Cardiology, St. Bartholomew's Hospital, London ECIA $7 \mathrm{BE}$. 